\title{
Cascaded KNN-BPN for Classification of Ears based on Shape Measures for Person Identification
}

\author{
Prashanth G.K. \\ Assistant Professor \\ Dept. of MCA, \\ Siddaganga Institute of Technology, Tumkur
}

\author{
M.A. Jayaram \\ Director, \\ Dept. of MCA, \\ Siddaganga Institute of Technology, Tumkur
}

\begin{abstract}
In this paper, a method to recognize persons using ear biometrics has been proposed. The recognition is through classification of Ears rendered conjointly by KNN and back propagation neural network. For this purpose, features of the Ear signifying its shape have been considered. To avail these features from 604 ear images, they were considered to be planar surfaces. Planar surface properties like the distribution of area, moment of inertia (MI) with respect to longest axis and MI with respect to an axis orthogonal to long axis and respective radii of gyration have been considered. KNN assisted BPN was able to categorize the ears into three distinct groups. The classification based person identification system showed an average recognition accuracy of $90 \%$. The other metrics like entropy, purity, precession, recall and f-measure showed a comparatively high value which is suggestive of adequate performance.
\end{abstract}

\section{Keywords}

BPN, Moment of Inertia, Radii of Gyration, Major Axis, Minor Axis, k-Means Clustering

\section{INTRODUCTION}

Alfred Iannarelli developed a new class of biometrics, based upon ear features and introduced it for use in the development of passive identification systems [1]. Identification by ear biometrics is promising because it is passive like face recognition. The ear is considered to be a unique feature for human beings. Even the ears of "identical twins" differ in some respects [2]. Unlike face, the configuration of ear will never be subjected to changes associated with changes in the facial expression, and the make-up effects. The configuration and the complexion of the ear do not vary with age. It has the biometric traits like uniqueness, universality, permanence and collectability.

Person identification is a problem of determining the identity of an individual by comparing a query image characteristic with subject images in the database. Biometric recognition of person is based on physiological or behavioural characteristics like ear, face, iris, fingerprint, signature, speech and gait. Human ears offer some distinct advantages over other biometric modalities: they have a wealthy of structural features that are permanent with increasing age from about 8 to 70 years old, and they are not affected by the expression variations. Current ear recognition approaches have exploited how to use $2 \mathrm{D}$ ear image and $3 \mathrm{D}$ ear model for human identification.

The ear can be used as a biometric system using a limited number of features. The decidability index of the ear is found to be more than that of the face but less than that of iris. The decidability index represents the separation of genuine and false scores for a biometric system. The characteristics responsible for making ear popular as a biometric system are given below:

- Ear has a uniform colour distribution; also it doesn't depend on expressions like face.

- Ear is easy to detect and localize as the background remains constant.

- Ear's size is much larger than other systems like Finger prints, iris, etc.

- It's not affected by cosmetics and spectacles.

- It's a passive trait and doesn't need cooperation of the person

The rest of the paper is organized as follows. Part II brings out the related works, part III elaborates shaped based biometric features used for the development of the model, part IV highlights the data, and its collection and associated image processing tasks. Part V describes the methodology used, the results and discussion are made in part VI, and the paper concludes in part VII.

\section{RELATED WORKS}

Though there are many reported applications of ANN in general and BPN in particular, the use of neural networks particular for classifications seems to be limited. Carmbola et al have developed a model for ear recognition in a security application. In this work, BPN is used along with wavelet transforms [3]. Classification is achieved by partitioning each pixel using [4]. This work is in connection with Irish images. They have used discriminatory information such as brightness local movements, local energy measurements and pixel location as inputs. Testing of the system showed an accuracy of $96 \%$ in determining which pixel in an image of eye is the pixel from the Iris. In a work related to face recognition system BPN is used along with PCA [5]. This work has three basic steps i. feature extraction, ii. Principal component analysis and iii. BPN for face recognition. Mai $\mathrm{V}$ et al [6] have proposed a new method to identify people using Electrocardiogram (ECG). QRS complex (Q waves, R waves, $S$ waves) which is a stable parameter against heart rate variability is used as a biometric feature. This work has reported for having achieved a classification accuracy of $97 \%$ using RBF.

Sulong et al [7] have used a combination of maximum pressure exerted on the keyboard and the time latency between the keystrokes to recognize the authenticate users and to reject imposters. In this work, RBFNN is used as a pattern 
matching method. The system so developed has been evaluated using False Reject Rate (FRR) and False Accept Rate (FAR). The researchers have affirmed the effectiveness of the security system designed by them.

Chatterjee et al [8] have proposed a new biometric system which is based on four types of temporal postural signals. The system employs $\mathrm{S}$-transform to determine the characteristic features for each human posture. An RBFNN with these characteristic features as input is developed for specific authentication. The training of the network has augmented extended Kalman filtering (EKF). The overall authentication accuracy of the system is reported to be of the order of $95 \%$.

In a study, multi-modal biometric consisting of fingerprint images and finger vein patterns were used to identify the authorized users after determining the class of users by RBFNN as a classifier. The parameters of the RBFNN were optimized using BAT algorithm. The performance of RBFNN was found to be superior when compared with KNN, Naïve Bayesian and non-optimized RBFNN classifier [9].

Ankit Chadha et al have used signature of persons for verification and authentication purpose. RBFNN was trained with sample images in the database. The network successfully identified the original images with the recognition accuracy of $80 \%$ for image sample size of 200 [10].

Handwriting recognition with features such as aspect ratio, end points, junction, loop, and stroke direction were used for recognition of writers [11]. The system used over 500 text lines from 20 writers. RBFNN showed a recognition accuracy of $95.5 \%$ when compared to back propagation network.

A novel approach directed towards the automatic clustering of $\mathrm{x}$-ray images has been attempted. The clustering was carried out based on multi-level feature of given x-ray images such as global level, local level and pixel level. The approach involves a combination of k-means and hierarchical clustering techniques this work has reported for having shown high level of accuracy [12].

$\mathrm{Xi}$ Cheng et al [13] have used similarity measures in multisample biometric systems. Both Pearson's correlation and Cosine similarity are used. Computational experiments have shown a better performance than using raw matching scores.

Roman V. et al [14] have compared performance of similarity measure functions to that obtained from customized fieldspecific approach in the domain of strategy-based behavioral biometrics. While all similarity measure functions showed a relatively high accuracy levels during user verification, weighted Euclidian similarity measures has slightly outperformed than general approaches such as Manhattan distance or Mahalanobis distance as claimed.

Satya Chaitanya Sripada et al [15] have compared the for Kmeans and Fuzzy $\mathrm{C}$ means clustering using the Purity and Entropy. The paper reported that, The K-means has lower value of purity and high value of entropy compared to Fuzzy $\mathrm{C}$ Means. The Fuzzy C means clustering is more accommodating for medical data sets when compared to $\mathrm{K}$ means.
Vikas Thada et al [16] have focused on comparative analysis for finding out the most relevant document for the given set of keywords by using three similarity measures viz Jaccard, Dice and Cosine similarity measures by using genetic algorithm approach. Due to the randomized nature of genetic algorithm the best fitness value is the average of 10 runs of the same code for a fixed number of iterations. The result states that the best fitness values were obtained using the Cosine similarity coefficients followed by Dice and Jaccard.

\section{SHAPE BASED BIOMETRICS}

In this work, the five shape based features of ears that were considered for classification are listed in the Table I. The details of feature extraction, their evaluation, authentication and development of an identification system is elaborated in seminal work of authors [17]. However, for the sake of completeness, the features are briefly explained in the following paragraphs.

The surface area of the ear is the projected area of the curved surface on a vertical plane. Moment of Inertia (MI) is the property of a planar surface which originates whenever one has to compute the moment of distributed load that varies linearly from the moment axis. Moment of Inertia is also viewed as a physical measure that signifies the shape of a planar surface and it is proved that by configuring the shape of planar surface and hence by altering the moment of inertia, the resistance of the planar surface against rotation with respect to a particular axis could be modulated or altered [18]. Therefore in this work, moment of inertia of ear surface with respect to two axes i.e. the major axis and the minor axis are considered to be the best biometric attributes that could capture the shape of irregular surface of the ear in a scientific way.

As far as features are concerned, major axis is the one which has the longest distance between the two points on the edge of the ear, the distance here is the maximum among point to point Euclidean distance. The minor axis is drawn in such way that it passes through tragus and is orthogonal to the major axis. Therefore, with different orientation of ears the orientation of major axis also changes. Being perpendicular to major axis, the orientation of minor axis is fixed.

The projected area is assumed to be formed out of segments. The area of an ear to the right side of the major axis is considered to be made out of six segments. Each of the segments thus subtends 300 with respect to the point of the intersection of the major axis and minor axis. The extreme edge of a sector is assumed to be a circular arc. Thus converting each segment into a sector of circle of varying area. Typical ear edge with measurements is shown in Figure 1.

\section{The measurements are}

$\theta$

$\rightarrow$ Inclination of the central radial axis of the segment with respect to minor axis (in degrees).

$r \rightarrow$ The length of the radial axis (in $\mathrm{mm}$ ).

The conversion of number of pixel into linear dimension (in $\mathrm{mm}$ ) was based on the resolution of the camera expressed in 
PPI (Pixel Per Inch). In this work 16Mega pixel camera, at 300 PPI was used. The computation of linear distance is straight farward $\mathrm{mm}=\left(\right.$ number of pixel $\left.^{*} 25.4\right) / \mathrm{PPI} \quad[1$ inch=25.4 mm]. With these measurements, the following parameters are computed.

Moment of inertia with respect to minor axis Imin

$$
I_{\min }=\sum_{i=1}^{6} a_{i} y_{i}^{2}
$$

Where ai is the area of a the ith segment and yi is the perpendicular distance of the centroid of the ith segment with respect to minor axis.

$$
\begin{gathered}
\mathrm{a}_{\mathrm{i}}=\Theta \mathrm{r}^{2} \\
\mathrm{y}_{\mathrm{i}}=\mathrm{C} \sin \theta
\end{gathered}
$$

Here, $\mathrm{C}$ is the centroidal distance of the segment with respect to the intersection point of the axes, which is given by[19];

$$
\mathrm{C}=\frac{2}{3} \frac{\mathrm{r} \sin \theta}{\theta}
$$

Simlarly, moment of inertia with respect to major axis Imax, $x i$ is the perpendicular distance of the centroid of the ith segment with respect to major axis.

$$
\begin{gathered}
I_{\max }=\sum_{i=1}^{6} a_{i} x_{i}^{2} \\
\text { Where } x_{i}=C \cos \theta
\end{gathered}
$$

From the computed values of moment of inertia and area of the ear surface, the radii of gyration with respect to minor axis (RGx) and major axis (RGy) were computed. The formulae for radii of gyration are given by [20].

$$
\begin{array}{r}
R G x=\sqrt{\frac{I_{\min }}{A}} \\
R G y=\sqrt{\frac{I_{\max }}{A}}
\end{array}
$$

Where, $\mathrm{A}$ is the sum of areas of six segments.

$$
\mathrm{A}=\sum_{\mathrm{i}=1}^{6} \mathrm{a}_{\mathrm{i}}
$$

Radius of gyration is the distance from an axis at which the mass of a body may be assumed to be concentrated and at which the moment of inertia will be equal to the moment of inertia of the actual mass about the axis. It is also equal to the square root of the quotient of the moment of inertia and the mass.

\begin{tabular}{cl}
\hline \multicolumn{2}{c}{ Table 1 Shape Based Features for Classification } \\
\hline Sl. No & Attributes \\
1 & Area (mm2) \\
2 & Moment of Inertia Y (Imax) (mm4) \\
3 & Radius of gyration Y (RGy) (mm) \\
4 & Moment of Inertia X (Imin) (mm4) \\
5 & Radius of gyration X (RGx) (mm) \\
\hline
\end{tabular}

\section{DATA FOR THE MODEL}

Ear images for this classification work were acquired from the pupils of Siddaganga group of institutes. The subjects involved were mostly students and faculty numbering 605 . In each acquisition session, the subject sat approximately one meter away with the side of the face in front of the camera in outside environment without flash. Ear is divided into 6 segments as seen in Figure 1.

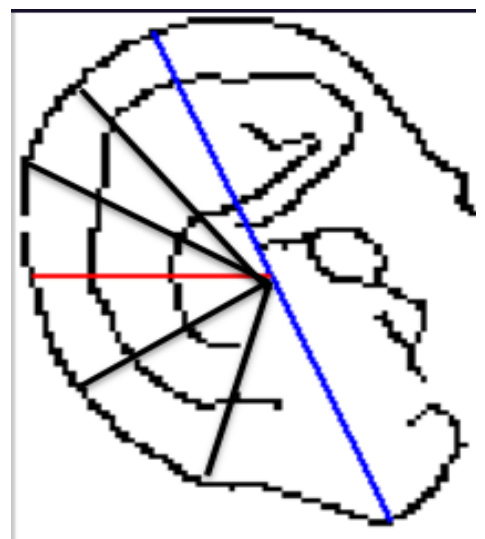

Figure 1. Outer edge of ear with major and minor axis

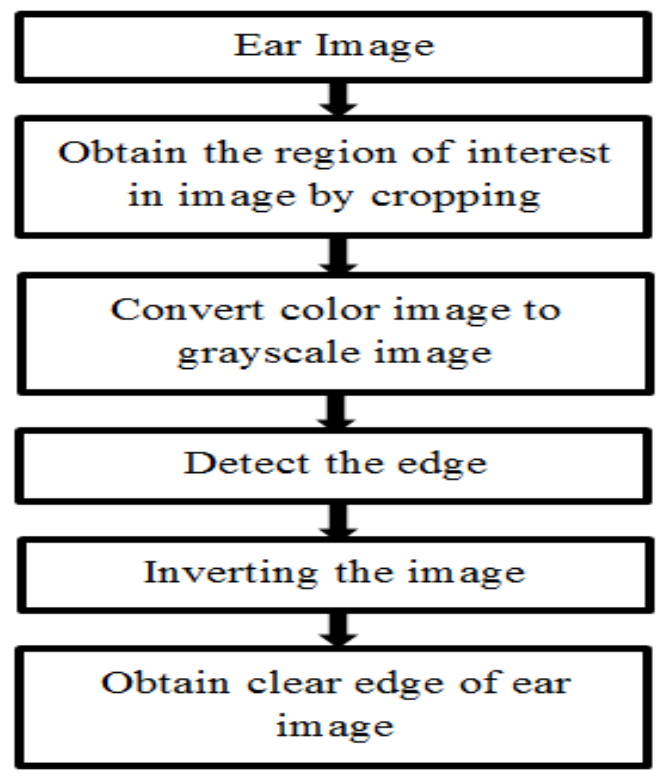

Figure 2: Steps involved in ear edge detection.

To isolate important and relevant information from the image, canny edge detection is used with threshold of 0.3 . Major and minor axes were identified. Major axis is the one which has the longest distance between two points on the edges of the ear. The minor axis is drawn in such a way that it passes through tragus and is orthogonal to the major axis.

\section{THE METHODOLOGY}

The methodology involved in this work includes two steps: i. Identify the optimum number of classes with minimum overlapping using KNN, and ii. Apply BPNN to fine tune the so obtained classes in step i. 


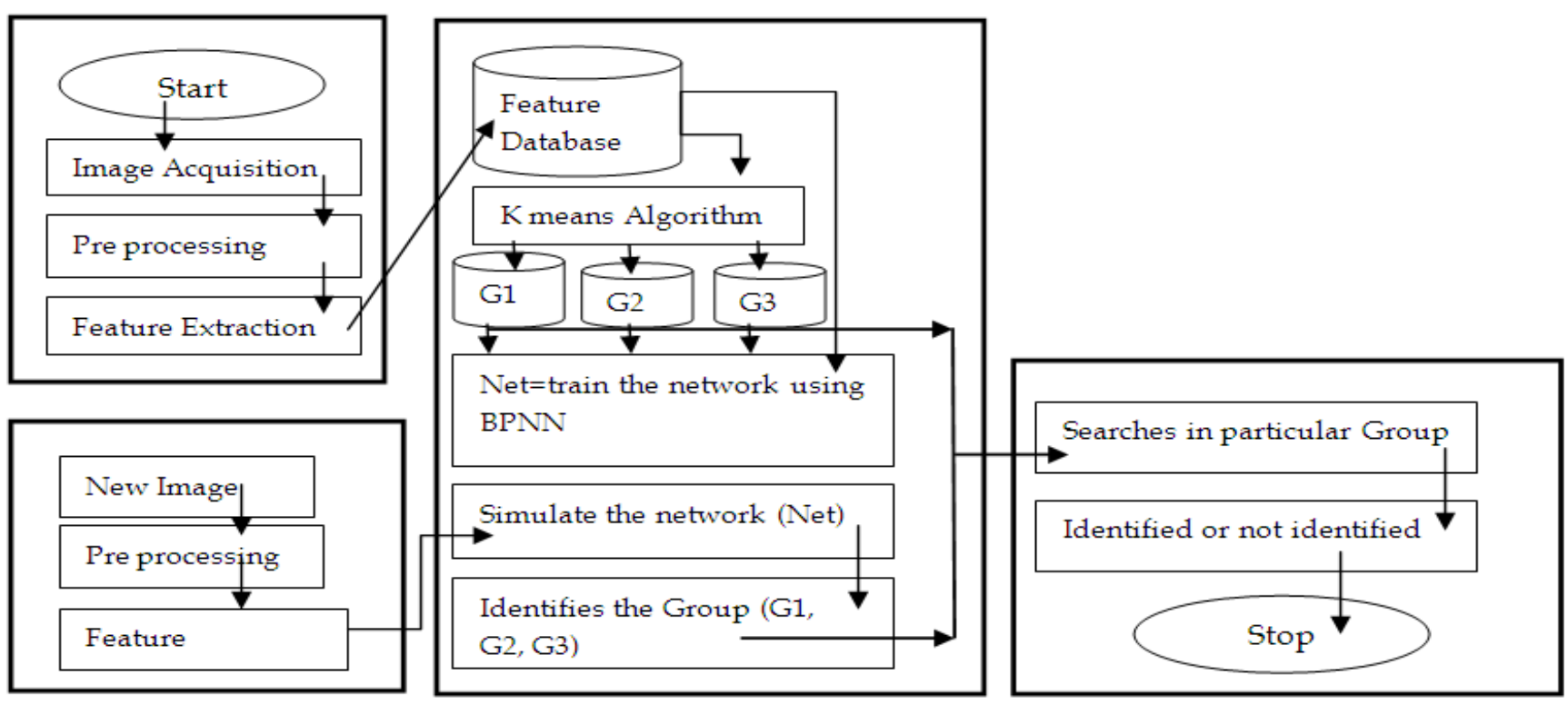

Figure 4: Flowchart of the work.

\subsection{K-means algorithm}

kmeans clustering is an iterative, data-partitioning algorithm that assigns $n$ observations to exactly one of $k$ clusters defined by centroids, where $\mathrm{k}$ is chosen before the algorithm starts. The purpose of applying the k-means clustering algorithm is to find a set of clustered centers and a partition of training data into subclasses. Normally, the center of each cluster is initialized to a randomly chosen input datum. Then each training datum is assigned to the cluster that is nearest to itself. After training data have been assigned to a new cluster unit, the new center of a cluster represents the average of the training data associated with that cluster unit. After all the new clusters have been calculated, the process is repeated until it converges [21]. The refined prototypic values of three groups after the successful execution of kmeans algorithm are presented in table 2 .

Series of computational experiments were conducted in order to find optimum number of classes with minimum overlapping. The experiments started with five, four and three classes. In all the cases the percentage of overlapping among the classes were verified. It was found that three groups were ideal because of minimum overlapping.

The centres of the groups so obtained are presented in Table 2 .

\begin{tabular}{cccccc}
\hline \multicolumn{6}{c}{ Table 2: Centroids of clusters as determined by K-means } \\
algorithm \\
\hline & Area & Imax & RGx & Imin & RGy \\
Group & 501.6093 & 6866031 & 118.308 & 1605.915 & 1.585156 \\
1 & & & & & \\
Group & 396.3764 & 3798362 & 98.40404 & 684.1802 & 1.156876 \\
2 \\
$\begin{array}{c}\text { Group } \\
3\end{array}$ & 270.4792 & 1666994 & 74.85014 & 292.8828 & 0.876999 \\
\hline
\end{tabular}

\subsection{Application of BPNN}

As a sequel to the first step, the data base was assorted into three groups. The sample segment of the database is shown in table 3. In the training mode of BPN $70 \%$ of the database of features values were selected from each of the groups. For this purpose a topology of the BPN has highlighted in the Table 3 was used.

The methodology used is conceptually shown in a block diagram depicted in Figure 4.

\begin{tabular}{ll}
\hline \multicolumn{2}{c}{ Table 4: Salient details of BPN implemented } \\
\hline No of input layer neurons & 5 \\
No of neurons in hidden layer & 20 \\
The basis function & sigmoid \\
Training function & trainlm \\
No of output neuron & 3 \\
\hline
\end{tabular}

The performance of BPN during the training and testing is highlighted in Table 5A and Table 5B.

\begin{tabular}{llll}
\hline \multicolumn{4}{c}{ Table 5A: Performance of BPN Training Mode } \\
\hline Classification & Data used & $\begin{array}{l}\text { Correctly } \\
\text { classified }\end{array}$ & $\begin{array}{l}\text { Percentage of } \\
\text { classification }\end{array}$ \\
Accuracy & & 74 & 93.70 \\
Group 1 & 79 & 231 & 93.10 \\
Group 2 & 248 & 119 & 93.00 \\
Group 3 & 128 & & \\
\hline
\end{tabular}

\begin{tabular}{|c|c|c|c|c|c|c|}
\hline \multicolumn{7}{|c|}{ Table 5B: Performance of BPN Testing Mode } \\
\hline \multicolumn{2}{|c|}{$\begin{array}{l}\text { Classification } \\
\text { Accuracy }\end{array}$} & $\begin{array}{l}\mathrm{Da} \\
\text { use }\end{array}$ & & $\begin{array}{l}\text { Correctly } \\
\text { classified }\end{array}$ & \multicolumn{2}{|c|}{$\begin{array}{l}\text { Percentage } \\
\text { of } \\
\text { classification }\end{array}$} \\
\hline \multicolumn{2}{|c|}{ Group 1} & \multicolumn{2}{|l|}{26} & 22 & \multicolumn{2}{|l|}{84.61} \\
\hline \multicolumn{2}{|c|}{ Group 2} & \multicolumn{2}{|l|}{82} & 76 & \multicolumn{2}{|c|}{92.68} \\
\hline \multicolumn{2}{|c|}{ Group 3} & \multicolumn{2}{|l|}{42} & 39 & \multicolumn{2}{|l|}{92.85} \\
\hline \multicolumn{7}{|c|}{ Table 3: Sample database of three groups } \\
\hline $\begin{array}{l}\text { Sl. } \\
\text { No }\end{array}$ & Area & $\operatorname{Imax}$ & RGy & $\operatorname{Imin}$ & RGx & Groups \\
\hline 1 & 131.3487 & 195355.9 & 38.56563 & 50.89822 & 0.622499 & 2 \\
\hline 2 & 135.0409 & 371368.5 & 52.44088 & 140.7316 & 1.020853 & 2 \\
\hline
\end{tabular}




\begin{tabular}{lllllll}
3 & 404.5635 & 3158077 & 88.35233 & 766.4084 & 1.376375 & 3 \\
4 & 241.7773 & 744226.9 & 55.48108 & 116.0455 & 0.692798 & 2 \\
5 & 370.4741 & 2691695 & 85.23815 & 310.3129 & 0.91521 & 2 \\
6 & 272.2138 & 3054815 & 105.9345 & 0.344449 & 0.035572 & 3 \\
7 & 358.0337 & 3395618 & 97.38621 & 254.3965 & 0.842934 & 3 \\
8 & 369.2937 & 2464924 & 81.69882 & 264.376 & 0.846107 & 2 \\
9 & 217.2377 & 2858884 & 114.7178 & 11.01563 & 0.225184 & 3 \\
10 & 360.2648 & 2844322 & 88.8543 & 641.5627 & 1.334469 & 3 \\
11 & 338.5039 & 1991710 & 76.70634 & 368.703 & 1.043654 & 2 \\
12 & 379.9424 & 5368573 & 118.8695 & 240.5883 & 0.795753 & 1 \\
13 & 412.1489 & 4025855 & 98.833 & 316.661 & 0.876537 & 3 \\
14 & 639.5815 & 9003344 & 118.6462 & 3243.304 & 2.251883 & 2 \\
15 & 376.3808 & 2785671 & 86.03025 & 647.0868 & 1.311196 & 2 \\
16 & 435.8933 & 4651435 & 103.3007 & 822.7214 & 1.37384 & 3 \\
17 & 369.8076 & 2818062 & 87.2946 & 258.6729 & 0.836349 & 3 \\
18 & 266.0732 & 1406845 & 72.71474 & 437.977 & 1.282995 & 2 \\
19 & 441.7652 & 7186791 & 127.5474 & 537.809 & 1.103363 & 1 \\
20 & 450.2947 & 4146650 & 95.96222 & 1295.909 & 1.696441 & 3 \\
21 & 405.7415 & 3655038 & 94.91202 & 237.0266 & 0.764318 & 3 \\
22 & 414.6593 & 4291438 & 101.7316 & 1138.657 & 1.657108 & 3 \\
23 & 439.04 & 5612504 & 113.0645 & 1007.022 & 1.514494 & 1 \\
24 & 569.4626 & 7267216 & 112.967 & 1253.937 & 1.483902 & 1 \\
25 & 388.5733 & 4062368 & 102.2476 & 810.9251 & 1.444621 & 3 \\
\hline & & & & & & \\
\hline
\end{tabular}

\section{RESULTS AND DISCUSSION}

The classification so rendered is evaluated by various metrics. TP is the number of correct predictions that an instance is negative.

- $\mathrm{FN}$ is the number of incorrect predictions that an instance is positive.

- FP is the number of incorrect of predictions that an instance negative and

- $\mathrm{TN}$ is the number of correct predictions that an instance is positive.

The Contingency Table 6. Is used to establish all the above said measures. The testing performance evaluation of BPN as shown by training accuracy, classification error and entropy is presented in Table 7. BPN in testing mode thus showed considerably a higher value of $88 \%$ of accuracy.

\begin{tabular}{lccc}
\hline \multicolumn{4}{c}{ Table 6: Contingency Table } \\
\hline & G1 & G2 & G3 \\
TP & 20 & 76 & 38 \\
TN & 03 & 02 & 01 \\
FP & 02 & 03 & 02 \\
FN & 01 & 01 & 01 \\
\hline
\end{tabular}

\begin{tabular}{ll}
\hline \multicolumn{2}{c}{ Table 7: Testing Performance Evaluation } \\
\hline Training accuracy & $88 \%$ \\
Classification Errors & 0.47333 \\
Entropy & 0.99 \\
\hline
\end{tabular}

\begin{tabular}{lrrr}
\hline \multicolumn{4}{c}{ Table 8: Group Analysis } \\
\hline G1 & G2 & \multicolumn{1}{l}{ G3 } \\
Accuracy & 0.884615 & 0.926829 & 0.928571 \\
Precision & 0.909091 & 0.962025 & 0.95 \\
Recall & 0.952381 & 0.987013 & 0.974359 \\
Specificity & 0.8 & 0.926829 & 0.904762 \\
F-measure & 0.930233 & 0.974359 & 0.962025 \\
\hline
\end{tabular}

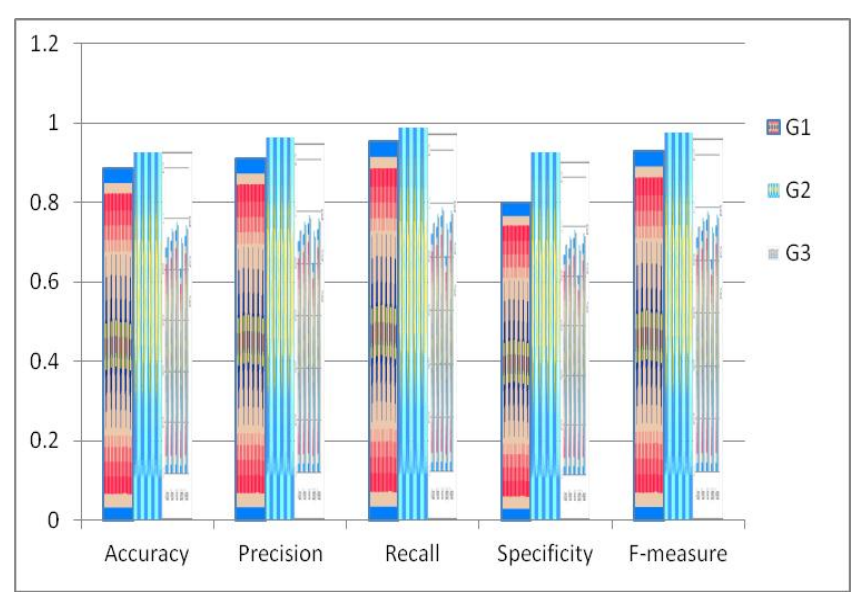

Figure 5: Bar chart of Group Analysis

A bar chart of the analysis of groups as elicited by BPN is shown in Figure 5. From this chart it is imperative that accuracy of the group varies from $88 \%$ to $92 \%$, the precision of all the groups is also considerable high, the groups have shown high recall, specificity and F-measures, and thus BPN proved to be an efficient classifier when it is guided by some a priory clustering algorithm like KNN.

\section{CONCLUSIONS}

This paper presented conjoint application of $\mathrm{KNN}$ and BPN in a cascaded fashion to classify ears based on novel shape based features. While KNN was used to find best possible number for groups with minimum overlapping, BPN was augmented to fine tune the classification task performed by KNN. The outcome of this work lies in appropriate merging of simple classification algorithms that can lead to robust grouping with generality.

\section{REFERENCES}

[1] Iannarelli, A., "Ear Identification", Forensic Identification Series, Paramount Publishing Company, Fremont, California (1989).

[2] Mahbubur Rahman et al, "Person Identification Using Ear Biometrics", International Journal of The Computer, the Internet and Management Vol. 15\#2 (2007) pp $1-8$.

[3] Samuel Adebayo Carambola Oladejo Daniel, Automatic Ear Recognition System using BPN,International Journal of Video and Image processing and network security, Vol 11, No 1, 2011.

[4] Randyi P, Broussard, Lauren R, Dlsoldion and Robert W U, Using "Artificial neural network and feature saliency techniques for improved Iris segmentation", Proceedings of international of joint conference on neural networks, orlando, Florida USA, August 12-17,2007 
[5] Mohammed Abdual Kashem, Md. Nasim Akthar, Shemi Ahamed, and Md. Mehbub alam, Face recognition system based on PCA and BPN, international journal of scientific and engineering research, Vol 2, Issue 6, June 2011.

[6] Mai V, Khalil I, Meli C, "ECG biometric using multilayer perceptron and radial basis function neural networks", 33rd Annual International Conference of the IEEE EMBS Boston, Massachusetts USA, August 30 September 3, 2011.

[7] A. Sulong , Wahyudi and M.D. Siddiqi, "Intelligent Keystroke Pressure-Based Typing Biometrics Authentication System Using Radial Basis Function Network", 2009 5th International Colloquium on Signal Processing \& Its Applications (CSPA).

[8] Chatterjee, A., Fournier, R., Nait-Ali, A., Siarry, P., "A Postural Information-Based Biometric Authentication System Employing S-Transform, Radial Basis Function Network, and Extended Kalman Filtering", Instrumentation and Measurement, IEEE Transactions on (Volume:59, Issue: 12 ).

[9] Anand Viswanathan, S. Chitra, "Optimized Radial Basis Function Classifier for Multi Modal Biometrics", Research Journal of Applied Sciences, Engineering and Technology 8(4): 521-529, 2014 ISSN: 2040-7459; eISSN: 2040-7467.

[10] Ankit Chadha, Neha Satam, Vibha Wali, "Biometric Signature Processing \& Recognition Using Radial Basis Function Network", CiiT International Journal of Digital Image Processing, ISSN 0974 - 9675 (Print) \& ISSN 0974 - 956X (Online) September 2013.

[11] Ashok.J, Rajan.E.G, (2010)"Writer Identification and Recognition Using Radial Basis Function", Int. Jour. of Computer Science and Information Technologies, 1(2), 51-57.

[12] Chhanda Ray, Krishnendu Sasmal, "A New Approach for Clustering of X-ray Images", IJCSI International
Journal of Computer Science Issues, Vol. 7, Issue 4, No 8, July 2010 ISSN (Online): 1694-0784 ISSN (Print): 1694-0814

[13] Xi cheng, Sergey Tulyakov and Venu Govindaraju, "Utilization of Matching Score vector Similarity Measures in Biometric Systems" , 978-1-4673-16125/12/\$31.00 2012 IEEE.

[14] Roman V, Yampolskiy and Venu Govindaraju, "Similarity Measure Function for Strategy-Based Biometrics", World Academy of science,Engineering and Technology 242006.

[15] Satya Chaitanya Sripada Dr. M.Sreenivasa Rao , "Comparison of purity and entropy of k-means clustering and fuzzy c means clustering", Indian Journal of Computer Science and Engineering (IJCSE).

[16] Vivek Jaglan, Vikas Thada, "Comparison of Jaccard, Dice, Cosine Similarity Coefficient To Find Best Fitness Value for Web Retrieved Documents Using Genetic Algorithm", published in International Journal of Innovations in Engineering and Technology (IJIET)

[17] M.A.Jayaram, Prashanth.G.K., Sachin.C.Patil,(2015) "Inertia based ear biometrics: A novel approach", Journal of Intelligent systems, De_Gruiter publishing, Germany, June 2015.

[18] Egor P Popov, "Engineering mechanics of solids, Easter economy edition, 2 nd edition 1998".

[19] B Arbab Zavar, Mark S Nixon, "Model based analysis for ear biometric", Computer Vision and Image understanding, vol 115, issue 4, P 487-502, 2011.

[20] Braja M. Das, Paul C. Hassler, "Statics and Mechanics of materials", Prentice Hall, 1988

[21] M C Natraj, M A Jayram ,C N Ravikumar ,Group Indexing fly ashes through radial basis function network, the Indian concert Journal July 2006. 